\title{
BINARY TREES, EXPLORATION PROCESSES, AND AN EXTENDED RAY-KNIGHT THEOREM
}

\author{
MAMADOU BA*** AND \\ ETIENNE PARDOUX, ${ }^{* * * *}$ Aix-Marseille Université \\ AHMADOU BAMBA SOW, ${ }^{* * * *}$ Université Gaston Berger
}

\begin{abstract}
We study the bijection between binary Galton-Watson trees in continuous time and their exploration process, both in the subcritical and in the supercritical cases. We then take the limit over renormalized quantities, as the size of the population tends to $\infty$. We thus deduce Delmas' generalization of the second Ray-Knight theorem.

Keywords: Galton-Watson process; Feller's branching; exploration process; Ray-Knight theorem

2010 Mathematics Subject Classification: Primary 60J80; 60F17

Secondary 92D25
\end{abstract}

\section{Introduction}

There are various forms of bijection between an exploration (or height) process and a random binary tree. This paper starts with a description of such a bijection, and a new rather simple proof that a certain law on the exploration paths is in bijection with the law of a continuous-time binary Galton-Watson random tree. The result in the critical case was first established by Le Gall [9], and in the subcritical case by Pitman and Winkel [10]; see also [6] and [8], where the exploration processes are jump processes, while ours are continuous. For similar results in the case where the approximating process is in discrete time and the tree is not necessarily binary, see [4]. We also consider the supercritical case, which is new. Inspired by the work of Delmas [3], we note that in the supercritical case, the random tree killed at time $a>0$ is in bijection with the exploration process reflected below $a$. Moreover, one can define a unique local time process, which describes the local times of all the reflected exploration processes, and has the same law as the supercritical Galton-Watson process. We then renormalize our Galton-Watson tree and height process, and take the weak limit, thus providing a new proof of Delmas' extension [3] of the second Ray-Knight theorem. The classical version of this theorem establishes the identity in law between the local time of reflected Brownian motion considered when the local time at 0 reaches $x$, at all levels, and a Feller critical branching diffusion. The same result holds in the subcritical and supercritical cases, with Brownian motion being replaced by Brownian motion with drift (in the supercritical case, reflection below an arbitrary level, as above, is needed). The exploration process in fact describes the genealogical tree (in the sense of [1]) of

\footnotetext{
Received 19 July 2010; revision received 12 October 2011.

* Postal address: Centre de Mathématiques et d'Informatique, LATP-CNRS, Aix-Marseille Université, 39 rue F. JoliotCurie, 13453 Marseille cedex 13, France.

** Email address: mba@cmi.univ-mrs.fr

*** Email address: pardoux@cmi.univ-mrs.fr

**** Postal address: LERSTAD, Université Gaston Berger, BP 234, Saint-Louis, Senegal.

Email address: ahmadou-bamba.sow@ugb.edu.sn
} 
the population, whose total mass follows a Feller stochastic differential equation (SDE). Our proof by approximation makes this interpretation completely transparent.

The paper is organized as follows. Section 2 is devoted to the description of the bijection between height curves and binary trees. In Section 3 we present the relation between the laws of height processes and Galton-Watson trees, and the 'discrete Ray-Knight theorem'. In Section 4 we present the results of convergence of both the population process and the height process, in the limit of large populations. Finally, in Section 5 we deduce the generalized Ray-Knight theorem from our convergences and the results at the discrete level.

\section{Preliminaries}

Fix $p>0$. Consider a continuous, piecewise-linear function $H$ from a subinterval of $\mathbb{R}_{+}$ into $\mathbb{R}_{+}$, which possesses the following properties: its slope is either $p$ or $-p$; it starts at time $t=0$ from 0 with the slope $p$; whenever $H(t)=0, H_{-}^{\prime}(t)=-p$ and $H_{+}^{\prime}(t)=p ; H$ is stopped at the time $T_{m}$ of its $m$ th return to 0 , which is supposed to be finite. We will denote by $\mathscr{H}_{p, m}$ the collection of all such functions. We will write $\mathscr{H}_{p}$ instead of $\mathscr{H}_{p, 1}$. We add the restriction that between two consecutive visits to 0 any function from $\mathscr{H}_{p, m}$ has all its local minima at distinct heights.

We denote by $\mathcal{T}$ the set of finite rooted binary trees which are defined as follows. An ancestor is born at time 0 . Until she eventually dies, she gives birth to an arbitrary number of offspring, but only one at a time. The same happens to each of her offspring, the offspring of her offspring, etc., until eventually the population dies out. We denote by $\mathcal{T}_{m}$ the set of forests which are the union of $m$ elements of $\mathcal{T}$.

There is a well-known bijection between binary trees and exploration processes. Under the curve representing an element $H \in \mathscr{H}_{p}$, we can draw a tree as follows. The height $h_{\text {lfmax }}$ of the leftmost local maximum of $H$ is the lifetime of the ancestor and the height $h_{\text {lowmin }}$ of the lowest nonzero local minimum is the time of the birth of the first offspring of the ancestor. If there is no such local minimum, the ancestor dies before giving birth to any offspring. We draw a horizontal line at level $h_{\text {lowmin }} H$ has two excursions above $h_{\text {lowmin }}$. The right excursion is used to represent the fate of the first offspring and her progeny. The left excursion is used to represent the fate of the ancestor and of the rest of her progeny, excluding the first offspring and her progeny. If there is no other local minimum of $H$ to the left or to the right of the first explored one, then there is no further birth: we draw a vertical line up to the unique local maximum, whose height is a death time. Continuing until there is no further local minimum/maximum to explore, this procedure defines a bijection $\Phi_{p}$ from $\mathscr{H}_{p}$ into $\mathcal{T}$ (see Figure 1). Repeating the same construction $m$ times, we extend $\Phi_{p}$ to a bijection between $\mathscr{H}_{p, m}$ and $\mathcal{T}_{m}$. Note that the horizontal distances between the vertical branches in the tree representation of the exploration process are arbitrary. See Figure 1(a).

We now define probability measures on $\mathscr{H}_{p}, \mathcal{T}$ and $\mathscr{H}_{p, m}, \mathcal{T}_{m}$. We first describe the subcritical case (by a slight abuse of terminology, subcritical in the present paper always means either subcritical or critical). Let $0<\mu \leq \lambda$ be two parameters. We define a stochastic process whose trajectories belong to $\mathscr{H}_{p}$ as follows. Let $\left\{U_{k}, k \geq 1\right\}$ and $\left\{V_{k}, k \geq 1\right\}$ be two mutually independent sequences of independent and identically distributed (i.i.d.) exponential random variables with means $1 / \lambda$ and $1 / \mu$, respectively. Let $Z_{k}=U_{k}-V_{k}, k \geq 1$. We denote by $\mathrm{P}_{\lambda, \mu}$ the law of the random element of $\mathscr{H}_{p}$, which is such that the height of the first local maximum is $U_{1}$ and that of the first local minimum is $\left(Z_{1}\right)^{+}$. If $\left(Z_{1}\right)^{+}=0$, the process is stopped. Otherwise, the height of the second local maximum is $Z_{1}+U_{2}$ and the height of the second local minimum is $\left(Z_{1}+Z_{2}\right)^{+}$, etc. Because $\mu \leq \lambda$, the process returns 


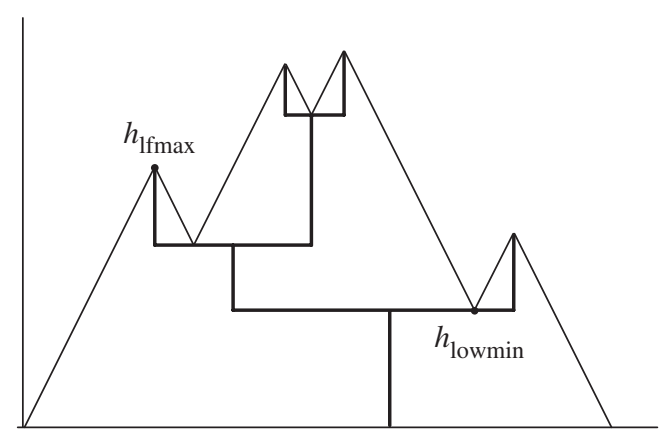

(a)

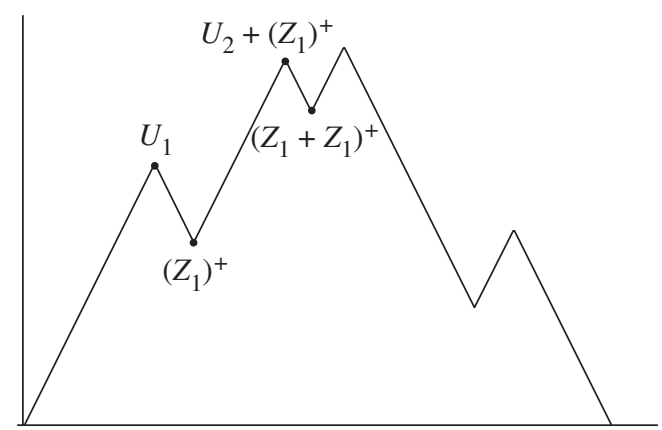

(b)

FIGURE 1: (a) Bijection between $\mathscr{H}_{2}$ and $\mathcal{T}$. (b) A trajectory of an exploration process.

to 0 almost surely (a.s.) in finite time. The random trajectory which we have constructed is an excursion above 0 (see Figure 1(b)). We similarly define a law on $\mathscr{H}_{p, m}$ as the concatenation of $m$ i.i.d. such excursions, and denote it by $\mathrm{P}_{\lambda, \mu}$. The random element defined above is called an exploration process or height process. We associate the continuous-time Galton-Watson tree (which is a random element of $\mathcal{T}$ ) with the same pair of parameters $(\lambda, \mu)$ as follows. The lifetime of each individual is exponential with expectation $1 / \lambda$, and during her lifetime, independently of it, each individual gives birth to offspring according to a Poisson process with rate $\mu$. The behaviors of the various individuals are i.i.d. We denote by $\mathrm{Q}_{\lambda, \mu}$ the law on $\mathcal{T}_{m}$ of a forest of $m$ i.i.d. random trees whose law is as just described.

In the supercritical case, i.e. $\lambda>\mu$, the exploration process defined above does not come back to 0 a.s. To overcome this difficulty, we add reflection below an arbitrary level $a>0$, and we consider the height process $H^{a}=\left\{H_{t}^{a}, t \geq 0\right\}$ reflected at level $a$ defined as above, with the addition of the rule that whenever the process reaches level $a$, it stops and starts immediately going down with slope $-p$ for an exponential duration of time with expectation $1 / \mu$. Again, the process stops when first going back to 0 . The reflected process $H^{a}$ comes back to 0 a.s. Indeed, let $A_{n}^{a}$ denote the event ' $H^{a}$ does not reach 0 during its $n$ first descents'. Clearly, we have, since the levels of local maxima are bounded by $a, \mathrm{P}\left(A_{n}\right) \leq(1-\exp (-\mu a))^{n}$, which goes to 0 as $n \rightarrow \infty$. Hence, the result. For each $a \in(0,+\infty)$, and any pair $(\lambda, \mu)$ of positive numbers, denote by $\mathrm{P}_{\lambda, \mu, a}$ the law of the process $H^{a}$. Define $\mathrm{Q}_{\lambda, \mu, a}$ to be the law of a binary 
Galton-Watson tree with birth rate $\mu$ and death rate $\lambda$, killed at time $t=a$ (i.e. all individuals alive at time $a^{-}$are killed at time $a$ ). The law $\mathrm{P}_{\lambda, \mu,+\infty}$ makes perfect sense in the case $\mu \leq \lambda$; $\mathrm{Q}_{\lambda, \mu,+\infty}$ is always well defined.

\section{Correspondence of laws}

The aim of this section is to prove that, for any $\lambda, \mu>0$ and $a \in(0,+\infty)$ (including the case $a=+\infty$ when $\mu \leq \lambda), \mathrm{P}_{\lambda, \mu, a} \Phi_{p}^{-1}=\mathrm{Q}_{\lambda, \mu, a}$. Let us state some basic results for homogeneous Poisson processes, which will be useful in the sequel.

\subsection{Preliminary results}

Let $T=\left(T_{k}\right)_{k \geq 0}$ be a Poisson point process on $\mathbb{R}_{+}$with intensity $\mu$. This means that $T_{0}=0$ and $\left(T_{k+1}-T_{k}, k \geq 0\right)$ are i.i.d. exponential random variables with mean $1 / \mu$. Let $\left(N_{t}, t \geq 0\right)$ be the counting process associated with $T$, that is, for all $t \geq 0$,

$$
N_{t}=\sup \left\{k \geq 0, T_{k} \leq t\right\}
$$

The following result is well known and elementary.

Lemma 3.1. Let $M$ be a nonnegative random variable independent of $T$, and define

$$
R_{M}=\sup _{k \geq 0}\left\{T_{k} ; T_{k} \leq M\right\}
$$

Then $M-R_{M} \stackrel{\mathrm{D}}{=} V \wedge M$, where $V$ and $M$ are independent, and $V$ has an exponential distribution with mean $1 / \mu$.

Moreover, on the event $\left\{R_{M}>s\right\}$, the conditional law of $N_{R_{M}^{-}}-N_{s}$ given $R_{M}$ is Poisson with parameter $\mu\left(R_{M}-s\right)$.

Lemma 3.2. Let $T=\left(T_{k}\right)_{k \geq 0}$ be a Poisson point process on $\mathbb{R}_{+}$with intensity $\mu$, and let $M$ be a positive random variable which is independent of $T$. Consider the integer-valued random variable $K$ such that $T_{K}=R_{M}$ and a second Poisson point process $T^{\prime}=\left(T_{k}^{\prime}\right)_{k \geq 0}$ with intensity $\mu$, which is jointly independent of the first and of $M$. Then $\bar{T}=\left(\bar{T}_{k}\right)_{k \geq 0}$, defined by

$$
\bar{T}_{k}= \begin{cases}T_{k} & \text { if } k<K \\ T_{K}+T_{k-K+1}^{\prime} & \text { if } k \geq K\end{cases}
$$

is a Poisson point process on $\mathbb{R}_{+}$with intensity $\mu$, which is independent of $R_{M}$.

Proof. Let $\left(N_{t}, t \geq 0\right),\left(\bar{N}_{t}, t \geq 0\right)$, and $\left(N_{t}^{\prime}, t \geq 0\right)$ be the counting processes associated to $T, \bar{T}$, and $T^{\prime}$.

It suffices to prove that, for any $n \geq 1,0<t_{1}<\cdots<t_{n}$, and $k_{1}, \ldots, k_{n} \in \mathbb{N}^{*}$,

$$
\xi_{t}=\mathrm{P}\left(\bar{N}_{t_{1}}=k_{1}, \ldots, \bar{N}_{t_{n}}=k_{n} \mid R_{M}\right)=\mathrm{e}^{-\mu t_{n}} \prod_{i=1}^{n} \frac{\left(\mu\left(t_{i}-t_{i-1}\right)\right)^{k_{i}-k_{i-1}}}{\left(k_{i}-k_{i-1}\right) !} .
$$

Since there is no harm in adding $t_{i} \mathrm{~s}$, we need only carry out the computation on the event that there exists $2 \leq i \leq n$ such that $t_{i-1}<R_{M}<t_{i}$, in which case a standard argument easily 
yields the claimed result, thanks to Lemma 3.1. Indeed, we have

$$
\begin{aligned}
\xi_{t}= & \mathrm{P}\left(N_{t_{1}}=k_{1}, \ldots, N_{t_{i-1}}=k_{i-1}, N_{R_{M}^{-}}+N_{t_{i}-t}^{\prime}=k_{i}, \ldots, N_{R_{M}^{-}}+N_{t_{n}-t}^{\prime}=k_{n}\right) \\
= & \mathrm{P}\left(N_{t_{1}}=k_{1}, \ldots, N_{t_{i-1}}-N_{t_{i-2}}=k_{i-1}-k_{i-2}, N_{R_{M}^{-}}-N_{t_{i-1}}+N_{t_{i}-R_{M}}^{\prime}=k_{i}-k_{i-1},\right. \\
& \left.\quad N_{t_{i+1}-R_{M}}^{\prime}-N_{t_{i}-R_{M}}^{\prime}=k_{i+1}-k_{i}, \ldots, N_{t_{n}-R_{M}}^{\prime}-N_{t_{n-1}-R_{M}}^{\prime}=k_{n}-k_{n-1}\right) \\
= & \mathrm{e}^{-\mu t_{n}} \prod_{i=1}^{n} \frac{\left(\mu\left(t_{i}-t_{i-1}\right)\right)^{k_{i}-k_{i-1}}}{\left(k_{i}-k_{i-1}\right) !} .
\end{aligned}
$$

\subsection{Basic theorem}

We are now in a position to prove the next theorem, which states a one-to-one correspondence between the tree associated with the exploration process $H^{a}$ defined in Section 2, and a continuous-time binary Galton-Watson tree with death rate $\lambda$ and birth rate $\mu$, killed at time $a$.

Theorem 3.1. For any $\lambda, \mu>0$ and $a \in(0,+\infty)$ (including the case $a=+\infty$ when $\mu \leq \lambda)$,

$$
\mathrm{Q}_{\lambda, \mu, a}=\mathrm{P}_{\lambda, \mu, a} \Phi_{p}^{-1}
$$

Proof. The individuals making up the population represented by the tree whose law is $\mathrm{Q}_{\lambda, \mu, a}$ are labeled $\ell=1,2, \ldots$, with individual 1 corresponding to the ancestor of the whole family. The subsequent individuals will be identified below. We will show that this tree is 'explored' by a process whose law is precisely $\mathrm{P}_{\lambda, \mu, a}$. We introduce the family $\left(T_{k}^{\ell}, k \geq 0, \ell \geq 1\right)$ of mutually independent Poisson processes with intensity $\mu$. For any $\ell \geq 1$, the process $T_{k}^{\ell}$ gives the birth times of the offspring of individual $\ell$. We define $U_{\ell}$ to be the lifetime of individual $\ell$.

Step 1. We start from the initial time $t=0$ and climb up to level $M_{1}$ of height $U_{1} \wedge a$, where $U_{1}$ follows an exponential law with mean $1 / \lambda$. We go down from $M_{1}$ until we find the most recent point of the Poisson process $\left(T_{k}^{1}\right)$, which gives the birth times of the offspring of individual 1. So, from Lemma 3.1, we have descended by $V_{1} \wedge M_{1}$, where $V_{1}$ follows an exponential law with mean $1 / \mu$, and is independent of $M_{1}$. We hence reach the level $m_{1}=M_{1}-V_{1} \wedge M_{1}$. If $m_{1}=0$, we stop; otherwise, we proceed to step 2 .

Step 2. We assign the label 2 to the last offspring of individual 1, born at time $m_{1}$. Let us define $\left(\bar{T}_{k}^{2}\right)$ by

$$
\bar{T}_{k}^{2}= \begin{cases}T_{k}^{1} & \text { if } k<K_{1}, \\ T_{K_{1}}^{1}+T_{k-K_{1}+1}^{2} & \text { otherwise }\end{cases}
$$

where $K_{1}$ is such that $T_{K_{1}}^{1}=m_{1}$.

Thanks to Lemma 3.2 , $\left(\bar{T}_{k}^{2}\right)$ is a Poisson process with intensity $\mu$ on $\mathbb{R}_{+}$, which is independent of $m_{1}$ and in fact also of $\left(U_{1}, V_{1}\right)$.

Starting from $m_{1}$, the exploration process climbs up to level $M_{2}=\left(m_{1}+U_{2}\right) \wedge a$, where $U_{2}$ is an exponential random variable with mean $1 / \lambda$, independent of $\left(U_{1}, V_{1}\right)$. Starting from level $M_{2}$, we go down a height $M_{2} \wedge V_{2}$, where $V_{2}$ follows an exponential law with mean $1 / \mu$ and is independent of $\left(U_{2}, U_{1}, V_{1}\right)$, to find the most recent point of the Poisson process $\left(\bar{T}_{k}^{2}\right)$. At this moment we are at the level $m_{2}=M_{2}-V_{2} \wedge M_{2}$. If $m_{2}=0$, we stop. Otherwise, we assign the label 3 to the individual born at time $m_{2}$, and repeat step 2 until we reach 0 . See Figure 2. 


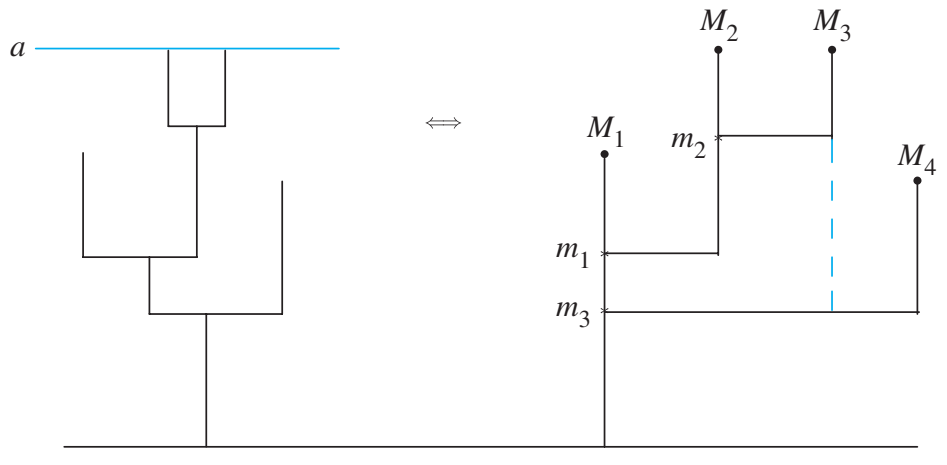

FIGURE 2: Two equivalent ways of representing a binary tree.

Since either we have a reflection at level $a$ or $\mu \leq \lambda, 0$ is reached a.s. after a finite number of iterations. It is clear that the random variables $M_{i}$ and $m_{i}$ fully determine the law $\mathrm{Q}_{\lambda, \mu, a}$ of the binary tree killed at time $t=a$, and they both have the same joint distribution as the levels of the successive local minima and maxima of the process $H^{a}$ under $\mathrm{P}_{\lambda, \mu, a}$.

\subsection{A discrete Ray-Knight theorem}

For any $a, \mu, \lambda>0$, we consider the exploration process $\left\{H_{t}^{a}, t \geq 0\right\}$ defined in Section 2 which is reflected in the interval $[0, a]$ and stopped at the first moment it reaches 0 for the $m$ th time. To this process, we can associate a forest of $m$ binary trees with birth rate $\mu$ and death rate $\lambda$, killed at time $t=a$, which all start with a single individual at the initial time $t=0$. Consider the branching process in continuous time $\left(Z_{t}^{a, m}, t \geq 0\right)$, which describes the number of offspring alive at time $t$ of the $m$ ancestors born at time 0 , whose progeny is killed at time $t=a$. Every individual in this population, independently of the others, lives for an exponential time with parameter $\lambda$ and gives birth to offspring according to a Poisson process of intensity $\mu$. We now choose the slopes of the piecewise-linear process $H^{a}$ to be \pm 2 (i.e. $p=2$ ). We define the local time accumulated by $H^{a}$ at level $t$ up to time $s$ as

$$
L_{s}^{a}(t)=\lim _{\varepsilon \downarrow 0} \frac{1}{\varepsilon} \int_{0}^{s} \mathbf{1}_{\left\{t \leq H_{r}^{a}<t+\varepsilon\right\}} \mathrm{d} r .
$$

Here $L_{s}^{a}(t)$ denotes the number of pairs of branches of $H^{a}$ which cross the level $t$ between times 0 and $s$. Note that a local minimum at level $t$ counts for two crossings, while a local maximum at level $t$ counts for no crossings. We have the following 'occupation time formula': for any integrable function $g$,

$$
\int_{0}^{s} g\left(H_{r}^{a}\right) \mathrm{d} r=\int_{0}^{\infty} g(r) L_{s}^{a}(r) \mathrm{d} r .
$$

Let

$$
\tau_{m}^{a}=\inf \left\{s>0: L_{s}^{a}(0) \geq m\right\} .
$$

Here $L_{\tau_{m}}^{a}(t)$ denotes the number of descendants of $m$ ancestors at time 0 , which are alive at time $t$. Then we have the following result. 
Lemma 3.3. For all $\lambda, \mu>0$ and $a \in(0,+\infty)$ (including the case $a=+\infty$ when $\mu \leq \lambda$ ),

$$
\left\{L_{\tau_{m}^{a}}^{a}(t), t \geq 0, m \geq 1\right\} \equiv\left\{Z_{t}^{a, m}, t \geq 0, m \geq 1\right\} \text { a.s. }
$$

We now want to establish a similar statement without the arbitrary parameter $a$. This is problematic only in the supercritical case. For any $0<a<b$, we define the application $\Pi^{a, b}$ which maps continuous trajectories with values in $[0, b]$ into trajectories with values in $[0, a]$ as follows. If $u \in C\left(\mathbb{R}_{+},[0, b]\right)$,

$$
\rho_{u}(s)=\int_{0}^{s} \mathbf{1}_{\{u(s) \leq a\}} \mathrm{d} r, \quad \Pi^{a, b}(u)(s)=u\left(\rho_{u}^{-1}(s)\right) .
$$

Lemma 3.4. It holds that

$$
\Pi^{a, b}\left(H^{b}\right) \stackrel{\mathrm{D}}{=} H^{a}
$$

Proof. It is in fact sufficient to show that the conditional law of the level of the first local minimum of $H^{b}$ after crossing the level $a$ downwards, given the past of $H^{b}$, is the same as the conditional law of the level of the first local minimum of $H^{a}$ after a reflection at level $a$, given the past of $H^{a}$. This identity follows readily from the 'lack of memory' of the exponential law.

Lemma 3.4 says that reflecting under $a$, or chopping out the pieces of the trajectory above level $a$, yields the same result (at least in law).

We now consider the $p=2$ case. To each $\lambda, \mu>0$ and $m \geq 1$, we associate the process $\left\{Z_{t}^{m}, t \geq 0\right\}$, which describes the evolution of the number of descendants of $m$ ancestors, with birth rate $\mu$ and death rate $\lambda$. For each $a>0$ (including the case $a=+\infty$ when $\mu \leq \lambda$ ), let $\left(H_{s}^{a}, s \geq 0\right)$ denote the exploration process of the genealogical tree of this population killed at time $a$, let $L^{a}$ denote its local time, and let $\tau_{m}^{a}$ be as defined in (3.1). It follows readily from Lemma 3.4 that, for any $0<a<b$,

$$
\left(L_{\tau_{m}^{b}}^{b}(t), 0 \leq t<a, m \geq 1\right) \stackrel{\mathrm{D}}{=}\left(L_{\tau_{m}^{a}}^{a}(t), 0 \leq t<a, m \geq 1\right)
$$

The compatibility relation (3.2) implies the existence of a projective limit $\left\{\mathcal{L}_{m}(t), t \geq 0\right.$, $m \geq 1$ \} with values in $\mathbb{R}_{+}$, which is such that, for each $a>0$,

$$
\left\{\mathcal{L}_{m}(t), 0 \leq t<a, m \geq 1\right\} \stackrel{\mathrm{D}}{=}\left\{L_{\tau_{m}^{a}}^{a}(t), 0 \leq t<a, m \geq 1\right\} .
$$

We have the following 'discrete Ray-Knight theorem'.

Proposition 3.1. It holds that

$$
\left\{\mathcal{L}_{m}(t), t \geq 0, m \geq 1\right\} \stackrel{\mathrm{D}}{=}\left\{Z_{t}^{m}, t \geq 0, m \geq 1\right\}
$$

Proof. It suffices to show that, for any $a \geq 0$,

$$
\left\{\mathcal{L}_{m}(t), 0 \leq t<a, m \geq 1\right\} \stackrel{\mathrm{D}}{=}\left\{Z_{t}^{m}, 0 \leq t<a, m \geq 1\right\}
$$

This result follows from (3.3) and Lemma 3.3. 


\subsection{Renormalization}

Let $x>0$ be arbitrary, and let $N \geq 1$ be an integer which eventually goes to $\infty$. Let $\left(Z_{t}^{[N x]}\right)_{t \geq 0}$ denote the branching process which describes the number of descendants at time $t$ of $[N x]$ ancestors in the population with birth rate $\mu_{N}=\sigma^{2} N / 2+\alpha$ and death rate $\lambda_{N}=$ $\sigma^{2} N / 2+\beta$, where $\alpha, \beta \geq 0$. We set

$$
X_{t}^{N, x}=\frac{Z_{t}^{[N x]}}{N}
$$

In particular, we have $X_{0}^{N, x}=[N x] / N \rightarrow x$ when $N \rightarrow+\infty$. Let $H^{a, N}$ be the exploration process associated to $\left\{Z_{t}^{[N x]}, 0 \leq t<a\right\}$, defined in the same way as previously, but with slopes $\pm 2 N$ and $\lambda, \mu$ replaced by $\lambda_{N}, \mu_{N}$. We also define $L_{S}^{a, N}(t)$, the local time accumulated by $H^{a, N}$ at level $t$ up to time $s$, as

$$
L_{s}^{a, N}(t)=\frac{4}{\sigma^{2}} \lim _{\varepsilon \downarrow 0} \frac{1}{\varepsilon} \int_{0}^{s} \mathbf{1}_{\left\{t \leq H_{r}^{a, N}<t+\varepsilon\right\}} \mathrm{d} r .
$$

The motivation of the factor $4 / \sigma^{2}$ will be clear after we have taken the limit as $N \rightarrow \infty$. $L_{s}^{a, N}(t)$ equals $4 / N \sigma^{2}$ times the number of pairs of $t$-crossings of $H^{a, N}$ between times 0 and $s$. Let

$$
\tau_{x}^{a, N}=\inf \left\{s>0: L_{s}^{a, N}(0) \geq \frac{4}{\sigma^{2}} \frac{[N x]}{N}\right\} .
$$

We define, for all $N \geq 1$, the projective limit $\left\{\mathcal{L}_{x}^{N}(t), t \geq 0, x>0\right\}$, which is such that, for each $a>0$,

$$
\left\{\mathcal{L}_{x}^{N}(t), 0 \leq t<a, x>0\right\} \stackrel{\mathrm{D}}{=}\left\{L_{\tau_{x}^{a, N}}^{a, N}(t), 0 \leq t<a, x>0\right\}
$$

Proposition 3.1 translates as follows.

Lemma 3.5. We have the identity in law

$$
\left\{\mathcal{L}_{x}^{N}(t), t \geq 0, x>0\right\} \stackrel{\mathrm{D}}{=}\left\{\frac{4}{\sigma^{2}} X_{t}^{N, x}, t \geq 0, x>0\right\} .
$$

\section{Weak convergence}

\subsection{Weak convergence of $X^{N, x}$}

The following result describes the limit of the sequence of processes $\left\{X^{N, x}, N \geq 1\right\}$; see, e.g. [7].

Proposition 4.1. We have $X^{N, x} \Rightarrow X^{x}$ as $N \rightarrow \infty$ for the topology of locally uniform convergence, where $X^{x}$ is the unique solution of the following Feller SDE:

$$
X_{t}^{x}=x+(\alpha-\beta) \int_{0}^{t} X_{r}^{x} \mathrm{~d} r+\sigma \int_{0}^{t} \sqrt{X_{r}^{x}} \mathrm{~d} B_{r}, \quad t \geq 0 .
$$




\subsection{Tightness criteria in $D([0,+\infty))$}

Let us present a sufficient condition for tightness which will be useful below. Consider a sequence $\left\{X_{t}^{n}, t \geq 0\right\}_{n \geq 1}$ of one-dimensional semimartingales, which is such that, for each $n \geq 1$,

$$
\begin{gathered}
X_{t}^{n}=X_{0}^{n}+\int_{0}^{t} \varphi_{s}^{n} \mathrm{~d} s+M_{t}^{n}, \quad 0 \leq t \leq T, \\
\left\langle M^{n}\right\rangle_{t}=\int_{0}^{t} \psi_{s}^{n} \mathrm{~d} s, \quad t \geq 0,
\end{gathered}
$$

where, for each $n \geq 1, M^{n}$ is a locally square-integrable martingale, and $\varphi^{n}$ and $\psi^{n}$ are progressively measurable processes with values in $\mathbb{R}$ and $\mathbb{R}_{+}$, respectively. Since our martingales $\left\{M_{t}^{n}, t \geq 0\right\}$ will be discontinuous, we need to consider their trajectories as elements of $D([0,+\infty))$, the space of right-continuous functions with left limits at every point, from $[0,+\infty)$ into $\mathbb{R}$, which we equip with the Skorokhod topology; see [2]. The following statement can be deduced from Theorems 13.4 and 16.10 of [2].

Proposition 4.2. A sufficient condition for the sequence $\left\{X_{t}^{n}, t \geq 0\right\}_{n \geq 1}$ to be tight in $D([0, \infty))$ is that both the sequence of random variables $\left\{X_{0}^{n}, n \geq 1\right\}$ is tight and, for some $c>0$,

$$
\sup _{n \geq 1, s>0}\left(\left|\varphi_{s}^{n}\right|+\psi_{s}^{n}\right) \leq c .
$$

If, moreover, for any $T>0$, as $n \rightarrow \infty$,

$$
\sup _{0 \leq t \leq T}\left|M_{t}^{n}-M_{t^{-}}^{n}\right| \rightarrow 0 \quad \text { in probability, }
$$

then any limit $X$ of a weakly converging subsequence of the original sequence $\left\{X^{n}\right\}_{n \geq 1}$ is a.s. continuous.

\subsection{Tightness of $\mathrm{H}^{a, N}$}

Consider now the exploration process $\left\{H_{s}^{a, N}, s \geq 0\right\}$ of the forest of trees representing the population $\left\{Z_{t}^{[N x]}, 0 \leq t<a\right\}$. Let $\left\{V_{s}^{a, N}, s \geq 0\right\}$ be the $\{-1,1\}$-valued process which is such that, $s$-almost everywhere, $\mathrm{d} H_{s}^{a, N} / \mathrm{d} s=2 N V_{s}^{a, N}$. The $\left(\mathbb{R}_{+} \times\{-1,1\}\right)$-valued process $\left\{\left(H_{s}^{a, N}, V_{s}^{a, N}\right), s \geq 0\right\}$ is a Markov process, which solves the SDE

$$
\begin{aligned}
& \frac{\mathrm{d} H_{s}^{a, N}}{\mathrm{~d} s}=2 N V_{s}^{a, N}, \quad H_{0}^{a, N}=0, \quad V_{0}^{a, N}=1, \\
& \mathrm{~d} V_{s}^{a, N}=2 \mathbf{1}_{\left\{V_{s^{-}}^{a, N}=-1\right\}} \mathrm{d} P_{s}^{+}-2 \mathbf{1}_{\left\{V_{s^{-}}^{a, N}=1\right\}} \mathrm{d} P_{s}^{-}+\frac{N \sigma^{2}}{2} \mathrm{~d} L_{s}^{a, N}(0)-\frac{N \sigma^{2}}{2} \mathrm{~d} L_{s}^{a, N}\left(a^{-}\right),
\end{aligned}
$$

where $\left\{P_{s}^{+}, s \geq 0\right\}$ and $\left\{P_{s}^{-}, s \geq 0\right\}$ are two mutually independent Poisson processes, with respective intensities

$$
\sigma^{2} N^{2}+2 \alpha N \text { and } \sigma^{2} N^{2}+2 \beta N,
$$

and $L_{s}^{a, N}(0)$ and $L_{s}^{a, N}\left(a^{-}\right)$respectively denote the number of visits to 0 and $a$ by the process $H^{a, N}$ up to time $s$, multiplied by $4 / N \sigma^{2}$ (see (3.4)). These two terms in the expression of $V^{a, N}$ stand for the reflection of $H^{a, N}$ above 0 and below $a$. Note that our definition of $L^{a, N}$ makes the mapping $t \rightarrow L_{s}^{a, N}(t)$ right continuous for each $s>0$. Hence, $L_{s}^{a, N}(t)=0$ for $t \geq a$, while $L_{s}^{a, N}\left(a^{-}\right)=\lim _{n \rightarrow \infty} L_{s}^{a, N}(a-1 / n)>0$ if $H^{a, N}$ has reached the level $a$ by time $s$. 
We now write a submartingale problem satisfied by the process $\left\{\left(H_{s}^{a, N}, V_{s}^{a, N}\right), s \geq 0\right\}$. We are not interested in writing it for arbitrary functions of the two variables $(h, v)$, but rather for specific functions, which will be convenient for taking the limit as $N \rightarrow \infty$. Note that the process $\left\{V_{s}^{a, N}, s \geq 0\right\}$ oscillates faster and faster as $N$ grows, and that in the limit some averaging takes place. We thus implement the so-called 'perturbed test function method' used in stochastic averaging; see e.g. [5]. For $f \in C^{2}(\mathbb{R})$, let

$$
\begin{gathered}
f^{N}(h, v)=f(h)+\frac{v}{N \sigma^{2}} f^{\prime}(h), \\
A^{N} f^{N}(h, v)=\frac{2}{\sigma^{2}} f^{\prime \prime}(h)+\mathbf{1}_{\{v=-1\}} \frac{4 \alpha}{\sigma^{2}} f^{\prime}(h)-\mathbf{1}_{\{v=+1\}} \frac{4 \beta}{\sigma^{2}} f^{\prime}(h) .
\end{gathered}
$$

If $f^{\prime}(0) \geq 0$ and $f^{\prime}(a) \leq 0$, then

$$
M_{s}^{f, N, a}:=f^{N}\left(H_{s}^{a, N}, V_{s}^{N}\right)-f^{N}(0,1)-\int_{0}^{s} A^{N} f^{N}\left(H_{r}^{N}, V_{r}^{N}\right) \mathrm{d} r
$$

is a local submartingale. If we choose successively $f(h)=h$ and $f(h)=h^{2}$, we deduce from (4.1) that there exist two local martingales $\left\{M_{s}^{1, a, N}, s \geq 0\right\}$ and $\left\{M_{s}^{2, a, N}, s \geq 0\right\}$ such that

$$
\begin{aligned}
H_{s}^{a, N}+\frac{V_{s}^{a, N}}{N \sigma^{2}}= & \frac{1}{N \sigma^{2}}+\frac{4 \alpha}{\sigma^{2}} \int_{0}^{s} \mathbf{1}_{\left\{V_{r}^{a, N}=-1\right\}} \mathrm{d} r-\frac{4 \beta}{\sigma^{2}} \int_{0}^{s} \mathbf{1}_{\left\{V_{r}^{a, N}=+1\right\}} \mathrm{d} r \\
& +\frac{1}{2}\left[L_{s}^{a, N}(0)-L_{0^{+}}^{a, N}(0)\right]-\frac{1}{2} L_{s}^{a, N}\left(a^{-}\right)+M_{s}^{1, a, N}
\end{aligned}
$$

and

$$
\begin{aligned}
\left(H_{s}^{a, N}\right)^{2}+\frac{2}{N \sigma^{2}} H_{s}^{a, N} V_{s}^{a, N}= & \frac{4}{\sigma^{2}} s+\frac{8 \alpha}{\sigma^{2}} \int_{0}^{s} \mathbf{1}_{\left\{V_{r}^{a, N}=-1\right\}} H_{r}^{a, N} \mathrm{~d} r \\
& -\frac{8 \beta}{\sigma^{2}} \int_{0}^{s} \mathbf{1}_{\left\{V_{r}^{a, N}=+1\right\}} H_{r}^{a, N} \mathrm{~d} r-a L_{s}^{a, N}\left(a^{-}\right)+M_{s}^{2, a, N} .
\end{aligned}
$$

It follows from the above computations that

$$
\left\langle M^{1, a, N}\right\rangle_{s}=\frac{4}{\sigma^{2}} s+\frac{8 \alpha}{N \sigma^{4}} \int_{0}^{s} \mathbf{1}_{\left\{V_{r}^{a, N}=-1\right\}} \mathrm{d} r+\frac{8 \beta}{N \sigma^{4}} \int_{0}^{s} \mathbf{1}_{\left\{V_{r}^{a, N}=1\right\}} \mathrm{d} r
$$

and from (4.3) that $\left\{M_{s}^{1, a, N}, s \geq 0\right\}$ is in fact a martingale. One difficulty which we want to eradicate is the additional complication of checking the tightness introduced by the local time terms in the expression for $H_{s}^{a, N}+V_{s}^{a, N} / N \sigma^{2}$. To this end, we consider a new pair of processes $\left(G^{a, N}, W^{a, N}\right)$, which is $(\mathbb{R} \times\{-1,1\})$-valued and satisfies

$$
\begin{aligned}
G_{s}^{a, N}=2 N \int_{0}^{s} W_{r}^{a, N} \mathrm{~d} r \\
W_{s}^{a, N}=1+\sum_{i \in \mathbb{Z}}\left\{2 \int_{0}^{s} \mathbf{1}_{\left\{a i \leq G_{r}^{a, N} \leq(i+1) a\right\}}(-1)^{i} \mathbf{1}_{\left\{W_{r^{-}}^{a, N}=-(-1)^{i}\right\}} \mathrm{d} P_{r}^{+}\right. \\
\left.\quad-2 \int_{0}^{s} \mathbf{1}_{\left\{a i \leq G_{r}^{a, N} \leq(i+1) a\right\}}(-1)^{i} \mathbf{1}_{\left\{W_{r^{-}}^{a, N}=(-1)^{i}\right\}} \mathrm{d} P_{r}^{-}\right\}
\end{aligned}
$$


with the same $P^{+}$and $P^{-}$as above. We claim that

$$
\begin{gathered}
H^{a, N}=\lim _{k \rightarrow \infty} \varphi_{k}\left(G^{a, N}\right) \quad \text { a.s., } \\
V^{a, N}=\sum_{i \in \mathbb{Z}}(-1)^{i} \mathbf{1}_{\left\{a i \leq G^{a, N} \leq(i+1) a\right\}} W^{a, N},
\end{gathered}
$$

where

$$
\varphi_{k}=\psi_{k} \circ \cdots \circ \psi_{1}
$$

and, for every $j$, the mapping $\psi_{j}$ from $\mathbb{R}$ into $\mathbb{R}$ is defined by

$$
\psi_{j}(x)= \begin{cases}|x| & \text { if } j \text { is odd } \\ a-|x-a| & \text { if } j \text { is even. }\end{cases}
$$

Indeed, since $G_{s}^{a, N}$ is locally bounded, to each $r>0$ we can associate a random index $k$ such that $0 \leq \psi_{k}\left(G_{s}^{a, N}\right) \leq a$ for $0 \leq s \leq r$, which implies that $\phi_{k+j}\left(G_{s}^{a, N}\right)=\phi_{k}\left(G_{s}^{a, N}\right)$ for $0 \leq s \leq r$ and $j \geq 1$. Note that $\psi_{1}$ and $\psi_{2}$ reflect the $G^{a, N}$ trajectory above 0 and below $a$, respectively. These operations are repeated until the thus obtained trajectory stays in $[0, a]$. We leave it to the reader to verify that it then coincides with $H^{a, N}$. Tightness of $\left\{G^{a, N}\right\}$ will imply that of $\left\{H^{a, N}\right\}$, since, for all $s, t$,

$$
\left|H_{s}^{a, N}-H_{t}^{a, N}\right| \leq\left|G_{s}^{a, N}-G_{t}^{a, N}\right| .
$$

We have

$$
\begin{aligned}
G_{s}^{a, N}+\frac{W_{s}^{a, N}}{N \sigma^{2}}= & \frac{1}{N \sigma^{2}}+\frac{4 \alpha}{\sigma^{2}} \sum_{i \in \mathbb{Z}} \int_{0}^{s} \mathbf{1}_{\left\{a i \leq G_{r}^{a, N} \leq(i+1) a\right\}}(-1)^{i} \mathbf{1}_{\left\{W_{r^{-}}^{a, N}=-(-1)^{i}\right\}} \mathrm{d} r \\
& -\frac{4 \beta}{\sigma^{2}} \sum_{i \in \mathbb{Z}} \int_{0}^{s} \mathbf{1}_{\left\{a i \leq G_{r}^{a, N} \leq(i+1) a\right\}}(-1)^{i} \mathbf{1}_{\left\{W_{r^{-}}^{a, N}=(-1)^{i}\right\}} \mathrm{d} r+\tilde{M}_{s}^{1, N, a}
\end{aligned}
$$

and

$$
\begin{aligned}
\left\langle\tilde{M}^{1, N, a}\right\rangle_{s}= & \frac{4}{\sigma^{2}} s+\frac{8 \alpha}{N \sigma^{4}} \sum_{i \in \mathbb{Z}} \int_{0}^{s} \mathbf{1}_{\left\{a i \leq G_{r}^{a, N} \leq(i+1) a\right\}} \mathbf{1}_{\left\{W_{r^{-}}^{a, N}=-(-1)^{i}\right\}} \mathrm{d} r \\
& +\frac{8 \beta}{N \sigma^{4}} \sum_{i \in \mathbb{Z}} \int_{0}^{s} \mathbf{1}_{\left\{a i \leq G_{r}^{a, N} \leq(i+1) a\right\}} \mathbf{1}_{\left\{W_{r^{-}}^{a, N}=(-1)^{i}\right\}} \mathrm{d} r .
\end{aligned}
$$

From (4.4), (4.5), and Proposition 4.2, tightness of the left-hand side of (4.4) follows. Since, moreover, $N^{-1} W_{s}^{a, N} \rightarrow 0$ a.s. uniformly with respect to $s$, the sequence $\left\{G^{a, N}, N \geq 1\right\}$ is tight in $D([0,+\infty))$. Because $G^{a, N}$ is a.s. continuous for each $N \geq 1$, Lemma 4.1 below follows from a well-known property of Skorokhod's topology.

Lemma 4.1. For any $a>0$, the sequence $\left\{H_{s}^{a, N}, s \geq 0\right\}_{N \geq 1}$ is tight in $C([0, \infty))$.

Remark 4.1. In the subcritical case $(\alpha \leq \beta)$, we can choose $a=+\infty$, which simplifies the above construction. We obtain $H^{N}$ from $G^{N}$ by reflection above $0\left(H^{N} \equiv\left|G^{N}\right|\right)$, and $G^{N}$ is defined by

$$
\begin{aligned}
G_{s}^{N} & =2 N \int_{0}^{s} W_{r}^{N} \mathrm{~d} r \\
W_{s}^{N} & =1+2 \int_{0}^{s} \operatorname{sgn}\left(G_{r}^{N}\right) \mathbf{1}_{\left\{W_{r^{-}}^{N}=-\operatorname{sgn}\left(G_{r}^{N}\right)\right\}} \mathrm{d} P_{r}^{+}-2 \int_{0}^{s} \operatorname{sgn}\left(G_{r}^{N}\right) \mathbf{1}_{\left\{W_{r^{-}}^{N}=\operatorname{sgn}\left(G_{r}^{N}\right)\right\}} \mathrm{d} P_{r}^{-} .
\end{aligned}
$$




\subsection{Weak convergence of $H^{a, N}$}

Let us state our convergence result.

Theorem 4.1. For any $a>0$ (including the case $a=+\infty$ when $\alpha \leq \beta$ ), $H^{a, N} \Rightarrow H^{a}$ in $C([0, \infty))$ as $N \rightarrow \infty$, where $\left\{H_{s}^{a}, s \geq 0\right\}$ is the process

$$
\frac{2(\alpha-\beta)}{\sigma^{2}} s+\frac{2}{\sigma} B_{s}
$$

reflected in $[0, a]$. In other words, $H^{a}$ is the unique weak solution of the reflected SDE

$$
H_{s}^{a}=\frac{2(\alpha-\beta)}{\sigma^{2}} s+\frac{2}{\sigma} B_{s}+\frac{1}{2} L_{s}(0)-\frac{1}{2} L_{s}\left(a^{-}\right) .
$$

The statement that $\left\{H_{s}^{a}, s \geq 0\right\}$ is the process $\left(2(\alpha-\beta) s / \sigma^{2}+2 B_{s} / \sigma, s \geq 0\right)$ reflected in $[0, a]$ amounts to saying (see $[11])$ that, for any $f \in C^{2}(\mathbb{R})$ with $f^{\prime}(0) \geq 0$ and $f^{\prime}(a) \leq 0$,

$$
M_{s}^{f}:=f\left(H_{s}^{a}\right)-f\left(H_{0}^{a}\right)-\frac{2}{\sigma^{2}} \int_{0}^{s}\left[f^{\prime \prime}\left(H_{r}^{a}\right)-(\alpha-\beta) f^{\prime}\left(H_{r}^{a}\right)\right] \mathrm{d} r
$$

is a submartingale. It remains to establish this property by taking the weak limit in (4.2). This will follow readily from the next result.

Lemma 4.2. For any sequence $\left(U^{N}, N \geq 1\right) \subset C([0,+\infty))$ which is such that $U^{N} \Rightarrow U$ as $N \rightarrow \infty$, and all $s>0$,

$$
\int_{0}^{s} \mathbf{1}_{\left\{V_{r}^{a, N}=1\right\}} U_{r}^{N} \mathrm{~d} r \Rightarrow \frac{1}{2} \int_{0}^{s} U_{r} \mathrm{~d} r, \quad \int_{0}^{s} \mathbf{1}_{\left\{V_{r}^{a, N}=-1\right\}} U_{r}^{N} \mathrm{~d} r \Rightarrow \frac{1}{2} \int_{0}^{s} U_{r} \mathrm{~d} r .
$$

Proof. It is an easy exercise to check that the mapping

$$
\Phi: C([0,+\infty)) \times C_{\uparrow}([0,+\infty)) \rightarrow C([0,+\infty))
$$

defined by

$$
\Phi(x, y)(t)=\int_{0}^{t} x(s) \mathrm{d} y(s)
$$

where $C_{\uparrow}([0,+\infty))$ denotes the set of increasing continuous functions from $[0, \infty)$ into $\mathbb{R}$ and the three spaces are equipped with the topology of locally uniform convergence, is continuous. Consequently, it suffices to prove that, locally uniformly in $s>0$,

$$
\int_{0}^{s} \mathbf{1}_{\left\{V_{r}^{a, N}=1\right\}} \mathrm{d} r \rightarrow \frac{s}{2}
$$

in probability as $N \rightarrow \infty$. In fact, since both the sequence of processes and the limit are continuous and monotone, it follows from the second Dini theorem that it suffices to prove the following result.

Lemma 4.3. For any $s>0$,

$$
\int_{0}^{s} \mathbf{1}_{\left\{V_{r}^{a, N}=1\right\}} \mathrm{d} r \rightarrow \frac{s}{2}
$$

in probability as $N \rightarrow \infty$. 
Proof. We have (the second line follows from (4.1))

$$
\begin{gathered}
\int_{0}^{s} \mathbf{1}_{\left\{V_{r}^{a, N}=1\right\}} \mathrm{d} r+\int_{0}^{s} \mathbf{1}_{\left\{V_{r}^{a, N}=-1\right\}} \mathrm{d} r=s, \\
\int_{0}^{s} \mathbf{1}_{\left\{V_{r}^{a, N}=1\right\}} \mathrm{d} r-\int_{0}^{s} \mathbf{1}_{\left\{V_{r}^{a, N}=-1\right\}} \mathrm{d} r=(2 N)^{-1} H_{s}^{a, N} .
\end{gathered}
$$

From Lemma 4.1, it readily follows that $(2 N)^{-1} H_{s}^{a, N} \rightarrow 0$ in probability as $n \rightarrow \infty$. We conclude by adding the two above identities.

Corollary 4.1. For each $a>0$ (including the case $a=+\infty$ when $\alpha \leq \beta$ ),

$$
\left(H^{a, N}, M^{1, N, a}, L_{.}^{a, N}(0), L_{.}^{a, N}\left(a^{-}\right)\right) \Rightarrow\left(H^{a}, \frac{2}{\sigma} B, L_{.}^{a}(0), L_{.}^{a}\left(a^{-}\right)\right),
$$

where $B$ is as above, and $L_{.}^{a}(0)$ and $L_{.}^{a}\left(a^{-}\right)$respectively denote the local time of the continuous semimartingale $H^{a}$ at level 0 and at level $a^{-}$.

Proof. Concerning the tightness, we only need to take care of the third and fourth terms in the quadruple. Consider the function $f^{N}(h, v)$ associated to some $f \in C^{2}(\mathbb{R})$ such that $f^{\prime}(0)=1$ and $f^{\prime}(a)=0$. We deduce, from (4.1),

$$
\begin{aligned}
L_{s}^{a, N}(0)= & 2 f\left(H_{s}^{a, N}\right)+2 \frac{V_{s}^{a, N}}{N \sigma^{2}} f^{\prime}\left(H_{s}^{a, N}\right)-2 f(0)-\frac{2}{N \sigma^{2}} f^{\prime}(0)-\frac{4}{\sigma^{2}} \int_{0}^{s} f^{\prime \prime}\left(H_{r}^{a, N}\right) \mathrm{d} r \\
& -\frac{8}{\sigma^{2}} \int_{0}^{s} f^{\prime}\left(H_{r}^{a, N}\right)\left(\alpha \mathbf{1}_{\left\{V_{r}^{N}=-1\right\}}-\beta \mathbf{1}_{\left\{V_{r}^{N}=1\right\}}\right) \mathrm{d} r-2 M_{s}^{f, N}-2 \tilde{M}_{s}^{f, N},
\end{aligned}
$$

where $M^{f, N}$ and $\tilde{M}^{f, N}$ are martingales such that

$$
\left\langle M^{f, N}\right\rangle_{s}=\frac{4}{\sigma^{2}} \int_{0}^{s}\left[f^{\prime}\left(H_{r}^{a, N}\right)\right]^{2} \mathrm{~d} r, \quad\left\langle\tilde{M}^{f, N}\right\rangle_{s} \leq \frac{c(f)}{N} s .
$$

Tightness of the local time terms follows from this formula (and a similar expression for $\left.L_{s}^{a, N}\left(a^{-}\right)\right)$. Then $\left(H^{a, N}, M^{1, N, a}, L^{a, N}(0), L^{a, N}\left(a^{-}\right)\right)_{N \geq 1}$ is tight in

$$
C([0, \infty)) \times[D([0, \infty))]^{3}
$$

Moreover, any weak limit of $M^{1, N, a}$ along a subsequence equals $2 B / \sigma$, since $\left\langle M^{1, N, a}\right\rangle_{s} \rightarrow$ $4 s / \sigma^{2}$ and the jumps of $M^{1, N, a}$ are equal in amplitude to $2 / N \sigma^{2}$. It then follows by taking the limit in (4.6) (and in a similar formula for $\left.L_{s}^{a, N}\left(a^{-}\right)\right)$that any weak limit of $\left(H^{a, N}, M^{1, N, a}\right.$, $\left.1 / 2 L_{s}(0), L^{a, N}\left(a^{-}\right)\right)$along a converging subsequence takes the form $\left(H^{a}, 2 B / \sigma, 1 / 2 L_{S}(0)\right.$, $\left.1 / 2 L_{s}\left(a^{-}\right)\right)$. Finally, from Theorem 5.7 of [11], the limit is unique; hence, the whole sequence converges.

\section{Generalized Ray-Knight theorem}

In this section we give an new proof of Delmas' generalization of the second Ray-Knight theorem. Define L.(0) to be the local time of $H$ at level 0 , and in the subcritical case $(\alpha \leq \beta)$

$$
\tau_{x}=\inf \left\{s>0, L_{s}(0)>\frac{\sigma^{2}}{4} x\right\} .
$$


In the supercritical case, of course the construction is more complex. It follows from Lemma 3.4 and Corollary 4.1 (see also Lemma 2.1 of [3]) that, for any $0<a<b$,

$$
\Pi^{a, b}\left(H^{b}\right) \stackrel{\mathrm{D}}{=} H^{a}
$$

where $H^{a}$ and $H^{b}$ are Brownian motion multiplied by $2 / \sigma$, with drift $2(\alpha-\beta) s / \sigma^{2}$, reflected in the intervals $[0, a]$ and $[0, b]$, respectively; see Theorem 4.1. Now define, for each $a, x>0$,

$$
\tau_{x}^{a}=\inf \left\{s>0, L_{s}^{a}(0)>\frac{4}{\sigma^{2}} x\right\} .
$$

It follows from (5.1) that, as in the discrete case, for all $0<a<b$,

$$
\left\{L_{\tau_{x}^{b}}^{b}(t), 0 \leq t<a, x>0\right\} \stackrel{\mathrm{D}}{=}\left\{L_{\tau_{x}^{a}}^{a}(t), 0 \leq t<a, x>0\right\} .
$$

Consequently, we can define the projective limit, which is a process $\left\{\mathcal{L}_{x}(t), t \geq 0, x>0\right\}$ such that, for each $a>0$,

$$
\left\{\mathcal{L}_{x}(t), 0 \leq t<a, x>0\right\} \stackrel{\mathrm{D}}{=}\left\{L_{\tau_{x}^{a}}^{a}(t), 0 \leq t<a, x>0\right\} .
$$

We have the following result (see Theorem 3.1 of [3]).

Theorem 5.1. (Generalized Ray-Knight theorem.) We have

$$
\left\{\mathcal{L}_{x}(t), t \geq 0, x>0\right\} \stackrel{\mathrm{D}}{=}\left\{\frac{4}{\sigma^{2}} X_{t}^{x}, t \geq 0, x>0\right\},
$$

where $X^{x}$, the Feller branching diffusion process, is the solution to the SDE

$$
X_{t}^{x}=x+(\alpha-\beta) \int_{0}^{t} X_{r}^{x} \mathrm{~d} r+\sigma \int_{0}^{t} \sqrt{X_{r}^{x}} \mathrm{~d} B_{r}, \quad t \geq 0 .
$$

Proof. Since both sides have stationary independent increments in $x$, it suffices to show that, for any $x>0$,

$$
\left\{\mathcal{L}_{x}(t), t \geq 0\right\} \stackrel{\mathrm{D}}{=}\left\{\frac{4}{\sigma^{2}} X_{t}^{x}, t \geq 0\right\} .
$$

Fix an arbitrary $a>0$. By applying the 'occupation time formula' to $H^{a, N}$, and Lemma 3.5, we have, for any $g \in C\left(\mathbb{R}_{+}\right)$with support in $[0, a]$,

$$
\frac{4}{\sigma^{2}} \int_{0}^{\tau_{x}^{a, N}} g\left(H_{r}^{a, N}\right) \mathrm{d} r=\int_{0}^{\infty} g(t) L_{\tau_{x}^{a, N}}^{a, N}(t) \mathrm{d} t \stackrel{\mathrm{D}}{=} \frac{4}{\sigma^{2}} \int_{0}^{\infty} g(t) X_{t}^{N, x} \mathrm{~d} t .
$$

We clearly deduce from Proposition 4.1 that

$$
\int_{0}^{\infty} g(t) X_{t}^{N, x} \mathrm{~d} t \Rightarrow \int_{0}^{\infty} g(t) X_{t}^{x} \mathrm{~d} t
$$

Let us admit for a moment that, as $N \rightarrow \infty$,

$$
\int_{0}^{\tau_{x}^{a, N}} g\left(H_{r}^{a, N}\right) \mathrm{d} r \Rightarrow \int_{0}^{\tau_{x}^{a}} g\left(H_{r}^{a}\right) \mathrm{d} r .
$$


From the occupation time formula for the continuous semimartingale $\left(H_{s}^{a}, s \geq 0\right)$, we have

$$
\frac{4}{\sigma^{2}} \int_{0}^{\tau_{x}^{a}} g\left(H_{r}^{a}\right) \mathrm{d} r=\int_{0}^{\infty} g(t) L_{\tau_{x}^{a}}^{a}(t) \mathrm{d} t .
$$

We deduce from (5.2), (5.3), (5.4), and (5.5) that, for any $g \in C\left(\mathbb{R}_{+}\right)$with compact support in $[0, a]$,

$$
\frac{4}{\sigma^{2}} \int_{0}^{\infty} g(t) X_{t}^{x} \mathrm{~d} t \stackrel{\mathrm{D}}{=} \int_{0}^{\infty} g(t) \mathcal{L}_{x}(t) \mathrm{d} t .
$$

In fact, this same argument can be slightly generalized, proving that, for any $n$ and any $g_{1}, \ldots, g_{n}$ with compact support, we have the following equality in distribution:

$$
\begin{aligned}
& \left(\frac{4}{\sigma^{2}} \int_{0}^{\infty} g_{1}(t) X_{t}^{x} \mathrm{~d} t, \ldots, \frac{4}{\sigma^{2}} \int_{0}^{\infty} g_{n}(t) X_{t}^{x} \mathrm{~d} t\right) \\
& \quad \stackrel{\mathrm{D}}{=}\left(\int_{0}^{\infty} g_{1}(t) \mathcal{L}_{x}(t) \mathrm{d} t, \ldots, \int_{0}^{\infty} g_{n}(t) \mathcal{L}_{x}(t) \mathrm{d} t\right) .
\end{aligned}
$$

Since both the processes $\left(X_{t}^{x}, t \geq 0\right)$ and $\left(\mathcal{L}_{x}(t), t \geq 0\right)$ are a.s. continuous, the theorem is proved.

It remains to prove (5.4), which is clearly a consequence of the following result (recall the definition of $\tau_{x}^{N}$ given in (3.5)).

Proposition 5.1. It holds that

$$
\left(H^{a, N}, \tau_{x}^{a, N}\right) \Rightarrow\left(H^{a}, \tau_{x}^{a}\right) .
$$

Proof. For the sake of simplifying the notation, we suppress the superscript $a$. Let us define the function $\phi$ from $\mathbb{R}_{+} \times C_{\uparrow}([0,+\infty))$ into $\mathbb{R}_{+}$by

$$
\phi(x, y)=\inf \left\{s>0: y(s)>\frac{4}{\sigma^{2}} x\right\} .
$$

For any fixed $x$, the function $\phi(x, \cdot)$ is continuous in the neighborhood of a function $y$ which is strictly increasing at the time when it first reaches the value $4 x / \sigma^{2}$. Define

$$
\tau_{x}^{\prime N}:=\phi\left(x, L_{.}^{N}(0)\right)
$$

We note that, for any $x>0, s \mapsto L_{s}(0)$ is a.s. strictly increasing at time $\tau_{x}$, which is a stopping time. This follows from the strong Markov property, the fact that $H_{\tau_{x}}=0$, and $L_{\varepsilon}(0)>0$ for all $\varepsilon>0$. Consequently, $\tau_{x}$ is a.s. a continuous function of the trajectory L.(0), and, from Corollary 4.1,

$$
\left(H^{N}, \tau_{x}^{\prime N}\right) \Rightarrow\left(H, \tau_{x}\right) .
$$

It remains to prove that $\tau_{x}^{\prime N}-\tau_{s}^{N} \rightarrow 0$ in probability. For any $y<x$ and large enough $N$,

$$
0 \leq \tau_{x}^{\prime N}-\tau_{x}^{N} \leq \tau_{x}^{\prime N}-\tau_{y}^{\prime N}
$$

Clearly, $\tau_{x}^{\prime N}-\tau_{y}^{\prime N} \Rightarrow \tau_{x}-\tau_{y}$; hence, for any $\varepsilon>0$,

$$
0 \leq \underset{N}{\lim \sup } \mathrm{P}\left(\tau_{x}^{\prime N}-\tau_{x}^{N} \geq \varepsilon\right) \leq \limsup _{N} \mathrm{P}\left(\tau_{x}^{\prime N}-\tau_{y}^{\prime N} \geq \varepsilon\right) \leq \mathrm{P}\left(\tau_{x}-\tau_{y} \geq \varepsilon\right) .
$$

The result follows, since $\tau_{y} \rightarrow \tau_{x^{-}}$as $y \rightarrow x, y<x$, and $\tau_{x^{-}}=\tau_{x}$ a.s. 


\section{Acknowledgements}

This work was partially supported by the ANR MANEGE (contract ANR-09-BLANC0215-03). The authors wish to thank the anonymous referee, whose excellent and detailed reports helped us to correct one error and significantly improve the presentation of our results.

\section{References}

[1] Aldous, D. (1991). The continuum random tree. I. Ann. Prob. 19, 1-28.

[2] Billingsley, P. (1999). Convergence of Probability Measures, 2nd edn. John Wiley, New York.

[3] Delmas, J.-F. (2008). Height process for super-critical continuous state branching process. Markov Process. Relat. Fields 14, 309-326.

[4] Duquesne, T. and Le Gall, J.-F. (2002). Random trees, Lévy processes and spatial branching processes. Astérisque 281, 147pp.

[5] Ethier, S. N. And Kurtz, T. G. (1986). Markov Processes. John Wiley, New York.

[6] Geiger, J. And Kersting, G. (1997). Depth-first search of random trees, and Poisson point processes. In Classical and Modern Branching Processes (IMA Vol. Math. Appl. 84), Springer, New York, pp. 111-126.

[7] Grimvall, A. (1974). On the convergence of sequences of branching processes. Ann. Prob. 2, 1027-1045.

[8] Lambert, A. (2010). The contour of splitting trees is a Lévy process. Ann. Prob. 38, 348-395.

[9] LE GALl, J.-F. (1989). Marches aléatoires, mouvement brownien et processus de branchement. In Séminaire de Probabilités XXIII (Lecture Notes Math. 1372), Springer, Berlin, pp. 258-274.

[10] Pitman, J. and Winkel, M. (2005). Growth of the Brownian forest. Ann. Prob. 33, 2188-2211.

[11] Stroock, D. W. and Varadhan, S. R. S. (1971). Diffusion processes with boundary conditions. Commun. Pure Appl. Math. 24, 147-225. 